\title{
Cavernous sinus involvement is not a risk factor for the primary tumor site treatment outcome of Sinonasal adenoid cystic carcinoma
}

Yi-Chan Lee ${ }^{1,7}$, Ta-Jen Lee ${ }^{2}$, Ngan-Ming Tsang ${ }^{3}$, Yenlin Huang ${ }^{5}$, Cheng-Lung Hsu' ${ }^{4}$ Li-Jen Hsin² ${ }^{2}$ Yi-Hsuan Lee ${ }^{6}$ and Kai-Ping Chang ${ }^{2,7^{*}}$

\begin{abstract}
Background: Sinonasal adenoid cystic carcinoma is a rare malignancy of the head and neck. Cavernous sinus invasion from sinonasal adenoid cystic carcinoma and its related management have rarely been investigated. This study evaluated the relationship between treatment outcome and cavernous sinus involvement in addition to other parameters.

Methods: A retrospective case series study was conducted at a tertiary referral center. The medical records of 47 patients diagnosed with primary sinonasal adenoid cystic carcinoma between 1984 and 2015 were retrospectively reviewed. The survival impact of the primary treatment modalities and the anatomic sites of tumor involvement were analyzed.

Results: Cavernous sinus invasion was observed in 8 patients (17\%), of whom 7 had ACC tumors originating from the maxillary sinus. The results of univariate analysis revealed that tumor stage, primary surgery, and the absence of skull-base and infratemporal fossa invasion were associated with better overall survival $(P=0.033, P=0.012, P=0.011$, and $P=0.040$, respectively) and better disease-free survival $(P=0.019, P=0.001, P=0.017$, and $P=0.029$, respectively). Multivariate analysis identified primary surgery as the only independent prognostic factor for disease-free survival $(P=0.026)$. Cavernous sinus invasion by sinonasal adenoid cystic carcinoma was not associated with worse overall survival or disease-free survival $(P=0.200$ and $P=0.198$, respectively).

Conclusions: Because maxillary adenoid cystic carcinoma is associated with a higher rate of cavernous sinus invasion, such cases warrant caution during preoperative planning. Primary surgery as the initial therapy provides better locoregional control and survival for patients with sinonasal adenoid cystic carcinoma. Cavernous sinus invasion did not significantly impact survival; thus, it should not be regarded as a contraindication for curative treatment.
\end{abstract}

Keywords: Sinonasal cancer, Adenoid cystic carcinoma, Cavernous sinus, Head and neck, Surgery, Chemoradiation

\footnotetext{
* Correspondence: dr.kpchang@gmail.com

${ }^{2}$ Department of Otolaryngology - Head and Neck Surgery, Chang Gung

Memorial Hospital, Taoyuan, Taiwan

${ }^{7}$ College of Medicine, Chang Gung University, Taoyuan, Taiwan

Full list of author information is available at the end of the article
} 


\section{Background}

Sinonasal malignant tumors are rare, accounting for approximately $3 \%$ of upper respiratory tract cancers. Adenoid cystic carcinoma (ACC), arising from the salivary glands, is the fourth most common malignant tumor in the sinonasal tract [1, 2]. Approximately $10-25 \%$ of ACC tumors in the head and neck region originate in the sinonasal region [3]. Some symptoms of sinonasal ACCs are similar to chronic sinusitis symptoms; thus, the diagnosis of sinonasal ACC is often delayed after initial presentation [3-5].

Previous studies have demonstrated that ACC is an aggressive tumor that spreads through either direct bone invasion or perineural invasion and frequently involves the skull base or intracranial region [3]. Cavernous sinus involvement of sinonasal ACC often occurs via perineural spread [6-10]; however, the incidence and treatment outcomes of cavernous sinus invasion by these tumors have never been addressed or analyzed. Hence, this study investigates the incidence and management of cavernous sinus invasion by sinonasal ACC in a single tertiary referral hospital. The primary outcome of this study is to clarify the prognosis of cavernous sinus involvement in patients with ACC upon presentation. Prognostic factors, treatment modalities, outcomes, and survival of patients with sinonasal ACC tumors were also analyzed.

\section{Methods}

This study enrolled patients who were diagnosed with sinonasal ACC between January 1984 and August 2015 at Chang Gung Memorial Hospital (Linko Medical Center, Taoyuan, Taiwan). Patients with at least one of the following conditions were excluded: prior history of malignancy, presence of a concomitant primary cancer (synchronous or metachronous), recurrent disease, or treatment by another hospital. Patients enrolled in this study had pathologically confirmed ACC tumors at one of the following sites: maxillary sinus, ethmoid sinus, sphenoid sinus, frontal sinus, or nasal cavity. ACC tumors originating from the nasopharynx were also excluded due to differences in staging and anatomical origin. The retrospective review of medical records for this study was approved by the Institutional Review Board (201600713B0).

Patients in the study received standard preoperative work-ups according to institutional guidelines, including a detailed history, complete physical examination, computed tomography or magnetic resonance imaging, chest radiographs, bone scan, and abdominal ultrasonography. Computed tomography or magnetic resonance imaging was used to determine the epicenter of the neoplasm. If the tumor involved multiple sites of the sinonasal region, the site with the greatest tumor volume was considered the epicenter. Tumor, node, and metastasis (TNM) staging was performed using the criteria for nasal cavity and paranasal sinus malignancy in the 2010 American Joint Committee on Cancer Staging Manual.

Medical records were reviewed for information regarding surgery, adjuvant therapy, radiotherapy, chemotherapy, recurrences, and follow-up period. If the primary treatment modality was surgery, the types of surgical resection included septectomy, maxillectomy, craniofacial resection, or endoscopic resection. Adjuvant therapy after surgery included radiotherapy or chemoradiotherapy. Chemoradiotherapy rather than surgical excision was used to treat cavernous sinus involvement. In patients who refused surgery as their primary treatment, radiotherapy (total target dose, at least 60 Gy delivered in fractions of 1.8-2 Gy per day for 5 days per week) was administered with or without concurrent chemotherapy (cisplatin-based regimens) [11]. All of the patients completed regular follow-up visits every 2 months for the first year after discharge, every 3 months for the second year, and every 6 months thereafter.

The associations between different categorical parameters were analyzed using the chi-square test or Fisher's exact test. All of the surviving patients received followups until November 2016 or the date of death. In terms of patient survival, the overall survival (OS) and diseasefree survival (DFS) rates were estimated using the Kaplan-Meier method and compared with the log-rank test. For OS, the event of interest was death from ACC directly or from an unrelated cause. For DFS, the event was death or any tumor relapse occurring loco-regionally or distantly. Multivariate regression analyses were applied to identify independent risk factors for OS and DFS. Statistical analyses were performed using PASW Statistics 18 (SPSS, Chicago, IL, USA). All $P$ values were two-sided with the significance level set at $P<0.05$.

\section{Results \\ Patient characteristics}

During the study period, 47 patients (20 females [42.6\%] and 27 males [57.4\%]) with sinonasal ACC tumors were enrolled (Table 1). The primary sites of sinonasal ACC were the maxillary sinus $(n=30)$, the ethmoid sinus $(n=8)$, and the nasal cavity $(n=9)$. Anatomic sites of ACC involvement were the cavernous sinus $(n=8$; $17.0 \%)$, the skull base $(n=11 ; 23.4 \%)$, infratemporal fossa $(n=18 ; 38.3 \%)$, and cheek skin $(n=7 ; 14.9 \%)$. Of the 8 patients with cavernous sinus invasion, 7 had maxillary and 1 had ethmoidal ACC. Tumor characteristics are described in Table 1.

\section{Treatment modality}

Surgical resection was the initial primary treatment in 30 patients with T1 $(n=6)$, T2 $(n=1)$, T3 $(n=7)$, T4a 
Table 1 Patient demographics and tumor stage

\begin{tabular}{|c|c|c|}
\hline Parameters & $\mathrm{N}$ & Percent \\
\hline All patients & 47 & 100 \\
\hline \multicolumn{3}{|l|}{ Sex } \\
\hline Male & 27 & 57.4 \\
\hline Female & 20 & 42.6 \\
\hline \multicolumn{3}{|l|}{ Age (yr) } \\
\hline Median & 52.4 & \\
\hline Range & $16.3-81.2$ & \\
\hline \multicolumn{3}{|l|}{ Follow-up (mo) } \\
\hline Median & 56.0 & \\
\hline Range & $3.7-326.5$ & \\
\hline \multicolumn{3}{|l|}{ Tobacco use } \\
\hline Never & 33 & 70.2 \\
\hline Former/current & 14 & 29.8 \\
\hline \multicolumn{3}{|l|}{ Alcohol use } \\
\hline Never & 36 & 76.6 \\
\hline Former/current & 11 & 23.4 \\
\hline \multicolumn{3}{|l|}{ T stage } \\
\hline $\mathrm{T} 1$ & 6 & 12.8 \\
\hline $\mathrm{T} 2$ & 1 & 2.1 \\
\hline T3 & 8 & 17.0 \\
\hline T4a & 19 & 40.4 \\
\hline T4b & 13 & 27.7 \\
\hline \multicolumn{3}{|l|}{ N stage } \\
\hline NO & 46 & 97.9 \\
\hline N1-3 & 1 & 2.1 \\
\hline \multicolumn{3}{|l|}{ M stage } \\
\hline MO & 46 & 97.9 \\
\hline M1 & 1 & 2.1 \\
\hline \multicolumn{3}{|l|}{ Clinical stage } \\
\hline 1 & 6 & 12.8 \\
\hline$\|$ & 1 & 2.1 \\
\hline III & 8 & 17.0 \\
\hline IVa & 18 & 38.3 \\
\hline $\mathrm{IVb}$ & 13 & 27.7 \\
\hline IVC & 1 & 2.1 \\
\hline
\end{tabular}

$(n=11)$, and T4b $(n=5)$ tumors. Of these 30 patients, 3 were treated with surgery alone, whereas the remaining 12 and 15 received adjuvant radiotherapy and chemoradiation therapy, respectively. Either chemoradiation or radiotherapy was the initial primary treatment for the remaining 17 patients with T3 $(n=1)$, T4a $(n=8)$, and T4b $(n=8)$ tumors. Of these 17 patients, 5 received radiotherapy alone, 11 received chemoradiotherapy, and 1 refused treatment due to his M1 status.

\section{Tumor relapse}

Fourteen patients developed recurrence (29.8\%), and the time from initial treatment to recurrence ranged from 15.7 to 257.7 months (median 53.6 months). The recurrence was local in 12 cases (25.5\%) and regional in 3 cases $(6.4 \%)$. Of the 14 patients with locoregional recurrence, $11(78.6 \%)$ had stage III or IV disease at initial presentation.

\section{Side effects and complications}

No severe complications were noted in the 8 patients with cavernous sinus invasion. Among the 8 patients, seven encountered temporary malaise with headache and were treated with dexamethasone. Many of them recovered, while 2 patients had trigeminal neuropathy (common toxicity criteria, grade 2), also regressing gradually with steroid use.

\section{Factors influencing survival}

The median time for OS and DFS was 77.1 months and 57.1 months, respectively. At 3, 5, and 10 years, the OS rates were $76.6 \%, 61.7 \%$, and $23.4 \%$, respectively, and the DFS rates were $66.0 \%, 44.7 \%$, and $14.9 \%$, respectively. In analyzing factors affecting survival during follow-up, patients with stage IV disease had significantly worse OS and DFS than patients with stage I, II, or III disease ( $P=0.026$ and $P=0.014$, respectively; Fig. $1 \mathrm{a}-\mathrm{b})$. OS and DFS were better in those who received surgery as the initial primary therapy than in those who received radiotherapy or chemoradiotherapy as the initial primary treatment $(P=0.009$ and $P<0.001$, respectively; Fig. 1a-b). Skull base invasion and infratemporal fossa invasion were specifically identified as negative factors affecting OS and DFS, whereas cavernous sinus invasion was not (Fig. 1a-b). In Fisher's exact test, no significant difference was found for either the local recurrence rate or the distant metastasis rate between patients with and without cavernous sinus invasion $(P=0.658, P=1.000$, respectively).

Patients who received surgery as the initial treatment included a group of 12 patients who received postoperative adjuvant radiotherapy and another group of 15 who received postoperative chemoradiation therapy. The differences in OS and DFS between these two groups were not significant $(P=0.118$ and $P=0.558$, respectively; Fig. 2a-b). The differences in OS and DFS between patients treated with radiotherapy alone and those treated with chemoradiation therapy alone were also not significant $(P=0.704$ and $P=0.977$, respectively; Fig. $2 \mathrm{a}-\mathrm{b}$ ).

To identify independent predictors of survival, multivariate analysis for OS and DFS was performed using age, sex, stage, skull base invasion, infratemporal fossa invasion, and cavernous sinus invasion as parameters in a Cox proportional regression model. Only surgery as 


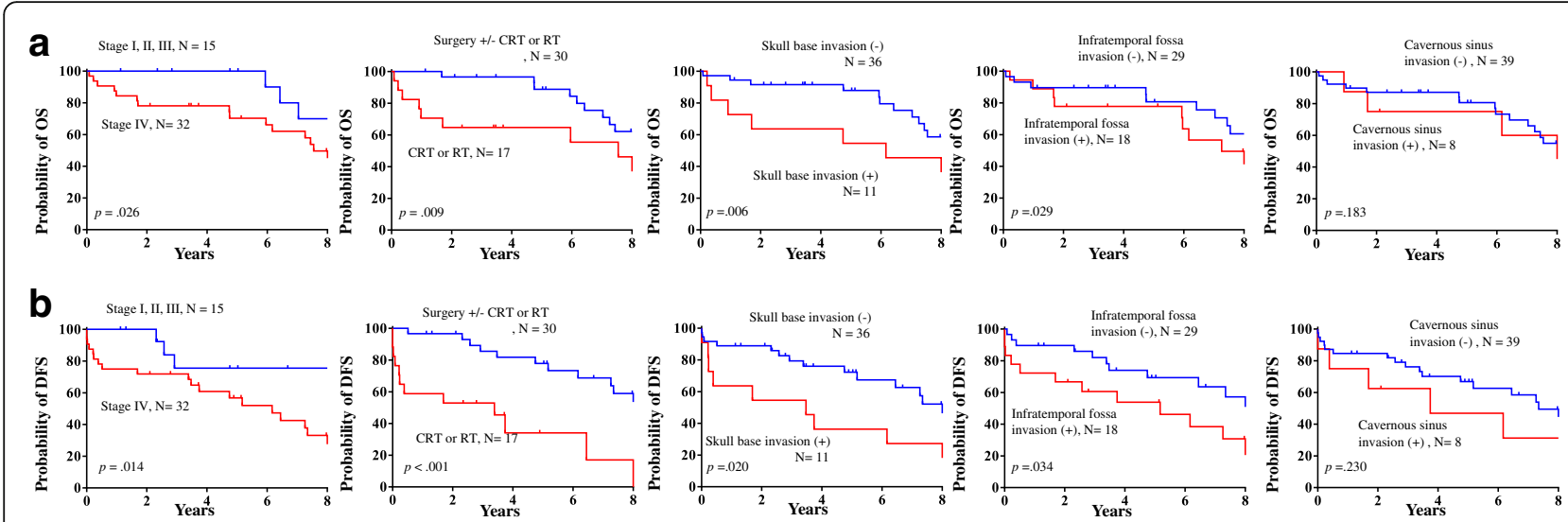

Fig. 1 a Kaplan-Meier curves of overall survival by tumor stage, primary surgery, skull base invasion, infratemporal fossa invasion and cavernous sinus invasion. b Kaplan-Meier curves of disease-free survival by tumor stage, primary surgery, skull base invasion, infratemporal fossa invasion and cavernous sinus invasion. CRT, chemoradiation therapy; RT, radiotherapy

the primary treatment was an independent factor influencing DFS (adjusted hazard ratio: 0.280 , 95\% confidence interval $=0.091-0.857, P=0.026$, Tables 2 and 3 ).

\section{Discussion}

In previous studies, most cases were predominantly stage IV because of the non-specific symptoms and late presentation of this rare malignancy and were associated with poor survival $[3,12-15]$. In this study, stage IV tumors were also diagnosed in most (68.1\%) patients, and those with stage IV disease exhibited a significantly worse OS. The primary treatment modalities for the original disease also affected survival outcome. KaplanMeier survival analysis showed that primary surgery as the initial treatment provided better OS and DFS, and the results of multivariate analysis indicated an association with a better DFS. A previous study demonstrated that the surgery type (endoscopic or external) was
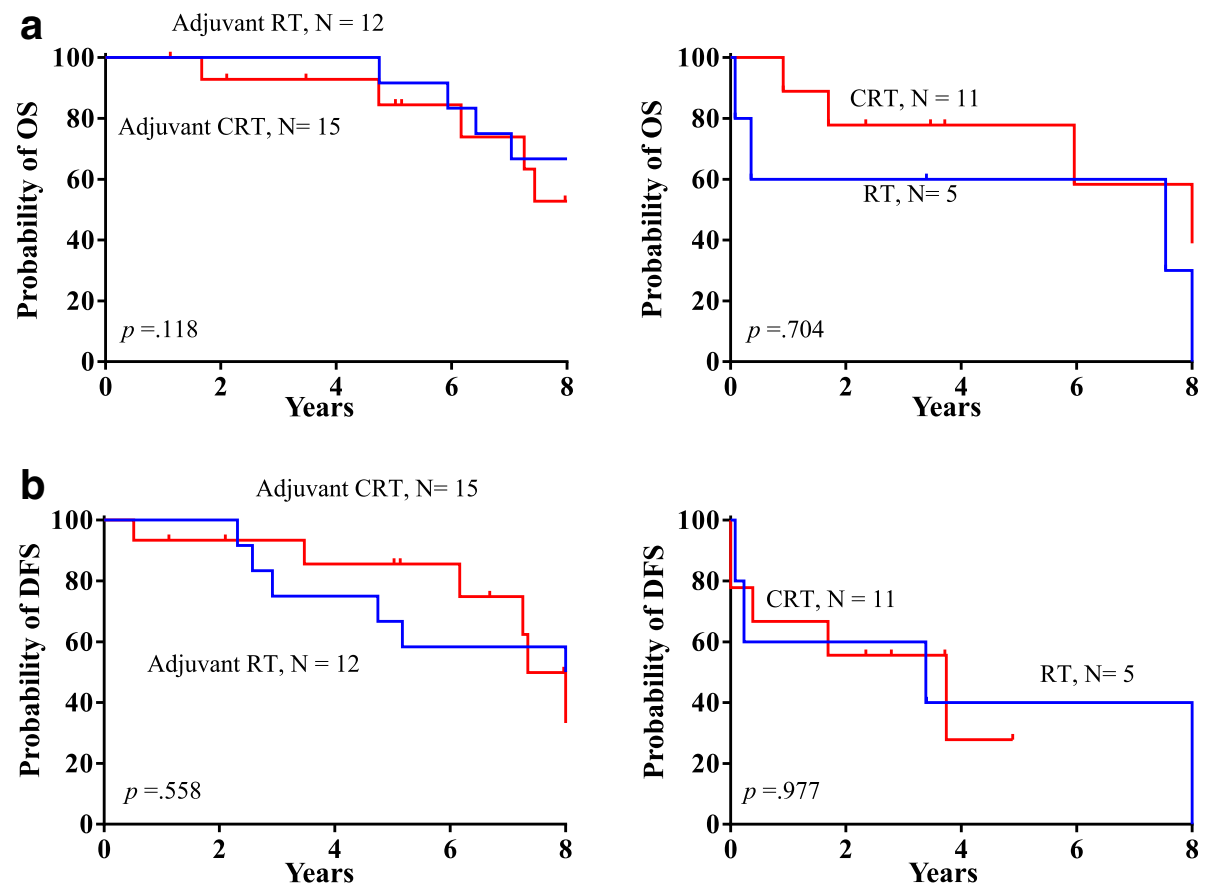

Fig. 2 a Kaplan-Meier curves of overall survival by postoperative adjuvant therapy and primary chemoradiation therapy compared with primary radiotherapy. $\mathbf{b}$ Kaplan-Meier curves of disease-free survival by postoperative adjuvant therapy and primary chemoradiation therapy compared with primary radiotherapy. CRT, chemoradiation therapy; RT, radiotherapy 
Table 2 Multivariate analysis of risk factors in relation to overall survival

\begin{tabular}{|c|c|c|c|c|}
\hline \multirow[b]{2}{*}{ Variables } & \multicolumn{2}{|l|}{ Univariate analysis } & \multicolumn{2}{|l|}{ Multivariate analysis } \\
\hline & $\begin{array}{l}\text { Hazard ratio } \\
(95 \% \mathrm{Cl})\end{array}$ & $P$-value & $\begin{array}{l}\text { Adjusted hazard ratio } \\
(95 \% \mathrm{Cl})\end{array}$ & $P$-value \\
\hline \multicolumn{5}{|l|}{ Age (yr) } \\
\hline$\leq 65$ & 1 & & 1 & \\
\hline$>65$ & $1.529(0.523-4.471)$ & 0.438 & $2.119(0.554-8.105)$ & 0.273 \\
\hline \multicolumn{5}{|l|}{ Sex } \\
\hline Female & 1 & & 1 & \\
\hline Male & $0.863(0.391-1.905)$ & 0.715 & $1.284(0.423-3.899)$ & 0.659 \\
\hline \multicolumn{5}{|c|}{ Clinical stage } \\
\hline I, II, III & 1 & & 1 & \\
\hline IV & $2.898(1.088-7.719)$ & $0.033^{*}$ & $2.397(0.612-9.386)$ & 0.209 \\
\hline \multicolumn{5}{|l|}{ Surgery } \\
\hline No & 1 & & 1 & \\
\hline Yes & $0.358(0.161-0.794)$ & $0.012^{*}$ & $0.690(0.247-1.927)$ & 0.479 \\
\hline \multicolumn{5}{|c|}{ Skull base invasion } \\
\hline No & 1 & & 1 & \\
\hline Yes & $3.054(1.290-7.233)$ & $0.011^{*}$ & $2.693(0.611-11.858)$ & 0.190 \\
\hline \multicolumn{5}{|c|}{ Infratemporal fossa invasion } \\
\hline No & 1 & & 1 & \\
\hline Yes & $2.414(1.041-5.600)$ & $0.040^{*}$ & $1.500(0.447-5.027)$ & 0.512 \\
\hline \multicolumn{5}{|c|}{ Cavernous sinus invasion } \\
\hline No & 1 & & 1 & \\
\hline Yes & $1.874(0.718-4.893)$ & 0.200 & $0.541(0.138-2.128)$ & 0.380 \\
\hline
\end{tabular}

Abbreviations: $\mathrm{Cl}$ confidence interval

*statistically significant

irrelevant if the tumor was completely resected [14]. Kaplan-Meyer analyses assessing the anatomic sites of tumor invasion both in this study and in a previous report found that skull base invasion and infratemporal fossa invasion negatively impacted survival $[3,16]$. Overall, for stage IV disease, the primary treatment modality (oncologically sound resection) and the anatomical location of tumor involvement were the major prognostic factors for this disease.

Winslow first referred to the small lateral sellar compartment as the "cavernous sinus." This pair of dural venous sinuses is a well-vascularized structure in the middle cranial fossa surrounded by the brain parenchyma [17]. The presence of cranial nerves III, IV, and V at the sinus capsule, in addition to cranial nerve VI, the internal carotid artery, and surrounding carotid sympathetic nerves, makes surgery at this location challenging. For malignant tumors involving the cavernous sinus, surgical removal of the cavernous sinus lesion is usually destructive and fraught with complications [18, 19]. Some authors have suggested limited excision of cavernous sinus tumors followed by adjuvant radiotherapy to treat the residual tumor; however, assessment of the oncological results for these treatments is not always feasible, and thus, the functional and oncological assessment of these approaches remains unclear [20, 21]. To avoid postoperative disability, we performed radical resection for sinonasal ACC, except when the tumor was involved in the cavernous sinus, and administered adjuvant chemoradiation therapy to the involved lesion. In addition, in seven of the 8 patients with cavernous sinus invasion, the primary site was the maxillary sinus. This may be due to a tendency for ACC tumors to invade perineurally; thus, the ACC tumors in the maxillary sinus would spread frequently through the maxillary branch of the trigeminal nerve. Consequently, in the pretreatment radiological work-ups for patients with maxillary ACC, extreme caution was advocated when examining the cavernous sinus. Among the 8 patients with cavernous sinus involvement, no recurrence was found. Furthermore, cavernous sinus invasion did not appear to cause tumor relapse or to be a negative prognostic factor in our univariate and multivariate analyses. Based on these findings, we suggest that the primary treatment modality 
Table 3 Multivariate analysis of risk factors in relation to disease-free survival

\begin{tabular}{|c|c|c|c|c|}
\hline \multirow[b]{2}{*}{ Variables } & \multicolumn{2}{|l|}{ Univariate analysis } & \multicolumn{2}{|l|}{ Multivariate analysis } \\
\hline & $\begin{array}{l}\text { Hazard ratio } \\
(95 \% \mathrm{Cl})\end{array}$ & $P$-value & $\begin{array}{l}\text { Adjusted hazard ratio } \\
(95 \% \mathrm{Cl})\end{array}$ & $P$-value \\
\hline \multicolumn{5}{|l|}{ Age (yr) } \\
\hline$\leq 65$ & 1 & & 1 & \\
\hline$>65$ & $1.545(0.528-4.520)$ & 0.427 & $1.670(0.600-4.649)$ & 0.326 \\
\hline \multicolumn{5}{|l|}{ Sex } \\
\hline Female & 1 & & 1 & \\
\hline Male & $1.036(0.477-2.250)$ & 0.929 & $1.841(0.494-6.857)$ & 0.363 \\
\hline \multicolumn{5}{|c|}{ Clinical stage } \\
\hline I, II, III & 1 & & 1 & \\
\hline IV & $3.334(1.217-8.129)$ & $0.019^{*}$ & $2.059(0.527-8.048)$ & 0.299 \\
\hline \multicolumn{5}{|l|}{ Surgery } \\
\hline No & 1 & & 1 & \\
\hline Yes & $0.231(0.100-0.536)$ & $0.001^{*}$ & $0.280(0.091-0.857)$ & $0.026^{*}$ \\
\hline \multicolumn{5}{|c|}{ Skull base invasion } \\
\hline No & 1 & & 1 & \\
\hline Yes & $2.750(1.200-6.302)$ & $0.017^{*}$ & $1.140(0.253-5.146)$ & 0.865 \\
\hline \multicolumn{5}{|c|}{ Infratemporal fossa invasion } \\
\hline No & 1 & & 1 & \\
\hline Yes & $2.251(1.098-5.789)$ & $0.029^{*}$ & $2.259(0.660-7.734)$ & 0.194 \\
\hline \multicolumn{5}{|c|}{ Cavernous sinus invasion } \\
\hline No & 1 & & 1 & \\
\hline Yes & $1.845(0.725-4.693)$ & 0.198 & $1.083(0.258-4.539)$ & 0.913 \\
\hline
\end{tabular}

Abbreviations: $\mathrm{Cl}$ confidence interval

*statistically significant

for sinonasal ACC with cavernous sinus invasion, similar to other sinonasal ACC tumors, should be surgery. Cavernous sinus invasion should not be considered a contraindication for surgery even if its involvement is not within the surgical field.

According to the literature reviewed, sinonasal ACC with skull base invasion has been mentioned to have a negative influence on survival [3]. In addition, infratemporal fossa invasion has also been associated with worse survival in the treatment of sinonasal malignancy [16]. Involvement of the cavernous sinus, on the other hand, has never been addressed before. The cavernous sinus is a small and complex anatomic structure with many cranial nerves passing through it, so invasion of the cavernous sinus often occurs due to the perineural spread and subsequently results in the apparent symptoms of cranial nerve palsy. The relatively early presentation of related symptoms may contribute to earlier intervention and management. Therefore, the authors assumed that the tumor burden of cavernous sinus invasion might be not usually too high to have a negative impact on survival.
Many studies have demonstrated that postoperative adjuvant radiotherapy can increase survival [3, 5, 15, 22]; however, chemotherapy has not been effective for treating sinonasal ACC $[3,23,24]$. In this study, we divided the 27 patients who received primary surgery into two groups, a postoperative adjuvant radiotherapy group $(n=12)$ and a postoperative adjuvant chemoradiotherapy group $(n=15)$, and observed no significant differences in DFS and OS between them. We also compared patients who received primary radiotherapy alone $(n=5)$ and patients who received primary chemoradiation therapy $(n=11)$ and found no significant difference in treatment outcomes. Based on these findings, the role of cisplatin-based chemotherapy in managing sinonasal ACC remains unclear and requires further study.

There are limitations in the present study. First, this is a retrospective study of a single tertiary referral center lending to potential bias. The relatively small sample size also renders these results somewhat arbitrary. However, sinonasal ACC is a rare disease entity, and cavernous sinus invasion caused by sinonasal 
ACC is even rarer. The present study attempted to add information to the current treatment of sinonasal ACC. A much larger study may be required to investigate the role of cavernous sinus invasion and different treatment modalities.

\section{Conclusions}

Cavernous sinus invasion was observed in approximately $17 \%$ of our patients with sinonasal ACC, and patients with maxillary ACC had a higher rate of cavernous sinus invasion. We found that survival was significantly improved among patients with sinonasal ACC tumors whose primary treatment was surgery. Although the cavernous sinus lesions in this series were treated solely by chemoradiotherapy, cavernous sinus invasion did not negatively impact survival outcomes in terms of OS and DFS. The role of cisplatin-based chemotherapy remains unclear after this retrospective investigation. The data suggest that primary surgery with adjuvant radiotherapy may provide a better treatment outcome for patients with sinonasal ACC, and cavernous sinus invasion is not a contraindication for curative treatment. Clinicians should remain alert to the possibility of cavernous sinus involvement in the pretreatment work-up and use caution when managing maxillary ACC patients.

\section{Abbreviations \\ ACC: Adenoid cystic carcinoma; DFS: Disease-free survival; OS: Overall Survival; TNM: Tumor, nodes, metastasis staging system}

\section{Acknowledgements}

Not applicable.

\section{Funding}

This study was supported by a grant (MOST105-2628-B-182A-008-MY3) from the Ministry of Science and Technology, a grant (CORPG3G0171) from Linko Chang Gung Memorial Hospital and a grant (CMRPG2G0231) from Keelung Chang Gung Memorial Hospital, Taiwan. The authors thank all the members of the Cancer Center at Chang Gung Memorial Hospital for their invaluable help.

\section{Availability of data and materials}

The datasets used and/or analyzed during the current study are available from the corresponding author on reasonable request.

\section{Authors' contributions}

YCL, TJL, NMT, YH, CLH, LJH, YHL and KPC all assisted in the preparation of this manuscript. Study design by $Y C L, N M T, Y H, C L H$ and KPC. Data collection by $Y C L, T J L, L H$ and $Y H L$. YHL and TJL prepare the abstract. Statistical analysis by $Y C L$ and KPC. All authors reviewed the finalized manuscript in preparation for submission. All authors read and approved the final manuscript.

\section{Ethics approval and consent to participate}

The retrospective review of medical records for this study was approved by the Institutional Review Board (201600713B0).

\section{Consent for publication}

Not applicable.

\section{Competing interests}

The authors declare that they have no competing interests.

\section{Publisher's Note}

Springer Nature remains neutral with regard to jurisdictional claims in published maps and institutional affiliations.

\section{Author details}

${ }^{1}$ Department of Otolaryngology - Head and Neck Surgery, Chang Gung Memorial Hospital, Keelung, Taiwan. ${ }^{2}$ Department of Otolaryngology - Head and Neck Surgery, Chang Gung Memorial Hospital, Taoyuan, Taiwan. ${ }^{3}$ Department of Radiation Oncology, Chang Gung Memorial Hospital, Taoyuan, Taiwan. ${ }^{4}$ Department of Hematology-Oncology, Chang Gung Memorial Hospital, Taoyuan, Taiwan. ${ }^{5}$ Department of Pathology, Chang Gung Memorial Hospital, Taoyuan, Taiwan. ${ }^{6}$ Department of Orthopedic Surgery, Buddhist Tzu-Chi General Hospital, Taipei, Taiwan. ${ }^{7}$ College of Medicine, Chang Gung University, Taoyuan, Taiwan.

Received: 15 November 2017 Accepted: 29 January 2018

Published online: 05 February 2018

\section{References}

1. Patel SH, Wang Z, Wong WW, Murad MH, Buckey CR, Mohammed K, et al. Charged particle therapy versus photon therapy for paranasal sinus and nasal cavity malignant diseases: a systematic review and meta-analysis. Lancet Oncol. 2014;15(9):1027-38. Epub 2014/07/02.

2. Robin TP, Jones BL, Gordon OM, Phan A, Abbott D, McDermott JD, et al. A comprehensive comparative analysis of treatment modalities for sinonasal malignancies. Cancer. 2017;123(16):3040-9. Epub 2017/04/04.

3. Lupinetti AD, Roberts DB, Williams MD, Kupferman ME, Rosenthal DI, Demonte F, et al. Sinonasal adenoid cystic carcinoma: the M. D. Anderson cancer center experience. Cancer. 2007;110(12):2726-31. Epub 2007/10/26.

4. Dulguerov P, Jacobsen MS, Allal AS, Lehmann W, Calcaterra T. Nasal and paranasal sinus carcinoma: are we making progress? A series of 220 patients and a systematic review. Cancer. 2001;92(12):3012-29. Epub 2001/12/26.

5. Rhee CS, Won TB, Lee CH, Min YG, Sung MW, Kim KH, et al. Adenoid cystic carcinoma of the sinonasal tract: treatment results. Laryngoscope. 2006; 116(6):982-6. Epub 2006/06/01.

6. Huang CC, Lee TJ. Radiology quiz case 2. Adenoid cystic carcinoma (ACC) of the sinonasal tract with perineural spread into the cavernous sinus. Arch Otolaryngol. 2008;134(9):1009-11. Epub 2008/09/17.

7. Ginsberg LE, Demonte F. Palatal adenoid cystic carcinoma presenting as perineural spread to the cavernous sinus. Skull Base Surg. 1998;8(1):39-43. Epub 2006/12/16.

8. Conde MA, Aladro Y, Villagra P. Perineural extension of a cylindroma causing a chronic cavernous sinus syndrome. Neurologia. 1998;13(9):445-6. Epub 1999/01/12. Extension perineural de un cilindroma originando un sindrome del seno cavernoso cronico.

9. Laccourreye O, Bely N, Halimi P, Guimaraes R, Brasnu D. Cavernous sinus involvement from recurrent adenoid cystic carcinoma. Ann Otol Rhinol Laryngol. 1994;103(10):822-5. Epub 1994/10/01.

10. Yamamoto T, Imai T. Adenoid cystic carcinoma presenting as cavernous sinus syndrome: a clinico-pathological study of two cases. No To Shinkei. 1989;41(5):519-25. Epub 1989/05/01.

11. Laurie $S A$, Ho AL, Fury MG, Sherman E, Pfister DG. Systemic therapy in the management of metastatic or locally recurrent adenoid cystic carcinoma of the salivary glands: a systematic review. Lancet Oncol. 2011;12(8):815-24. Epub 2010/12/15.

12. Seong SY, Hyun DW, Kim YS, Cho HJ, Lee JG, Yoon JH, et al. Treatment outcomes of sinonasal adenoid cystic carcinoma: 30 cases from a single institution. Journal Craniomaxillofac Surg. 2014;42(5):e171-5. Epub 2013/10/02.

13. Unsal AA, Chung SY, Zhou AH, Baredes S, Eloy JA. Sinonasal adenoid cystic carcinoma: a population-based analysis of 694 cases. Int Forum Allergy Rhinology. 2017;7(3):312-20. Epub 2016/11/20.

14. Michel J, Fakhry N, Santini L, Mancini J, Giovanni A, Dessi P. Sinonasal adenoid cystic carcinomas: clinical outcomes and predictive factors. Int J Oral Maxillofac Surg. 2013;42(2):153-7. Epub 2012/12/12.

15. Michel G, Joubert M, Delemazure AS, Espitalier F, Durand N, Malard O. Adenoid cystic carcinoma of the paranasal sinuses: retrospective series and review of the literature. Eur Ann Otorhinolaryngol Head Neck Dis. 2013; 130(5):257-62. Epub 2013/06/12.

16. Madani I, Bonte K, Vakaet L, Boterberg T, De Neve W. Intensity-modulated radiotherapy for sinonasal tumors: Ghent University Hospital update. Int J Radiat Oncol Biol Phys. 2009;73(2):424-32. Epub 2008/08/30. 
17. Winslow JB, Simonneau C. In: Desessartz J, editor. Exposition anatomique de la structure du corps humain: chez Guillaume Desprez; 1732.

18. Jimbo H, Kamata S, Miura K, Asamoto S, Tada S, Endo T, et al. Operative management of skull base malignant tumors arising from the nasal cavity and paranasal sinus: recent strategies used in 25 cases. Neurol Med Chir. 2010;50(1):20-6. discussion 6. Epub 2010/01/26.

19. Morimoto K, Demizu Y, Hashimoto N, Mima M, Terashima K, Fujii O, et al. Particle radiotherapy using protons or carbon ions for unresectable locally advanced head and neck cancers with skull base invasion. Jpn J Clin Oncol. 2014;44(5):428-34. Epub 2014/03/13.

20. Chen L, Huang X, Mao Y, Zhou L. Reconsideration of cavernous sinus surgeries. Neurosurgery. 2014;61(Suppl 1):130-4. Epub 2014/07/18.

21. Ramakrishna R, Raza SM, Kupferman M, Hanna E, DeMonte F. Adenoid cystic carcinoma of the skull base: results with an aggressive multidisciplinary approach. J Neurosurg. 2016;124(1):115-21. Epub 2015/08/08.

22. Husain Q, Kanumuri W, Svider PF, Radvansky BM, Boghani Z, Liu JK, et al. Sinonasal adenoid cystic carcinoma: systematic review of survival and treatment strategies. Otolaryngology Head Neck Surg. 2013;148(1):29-39. Epub 2012/10/16.

23. Papaspyrou G, Hoch S, Rinaldo A, Rodrigo JP, Takes RP, van Herpen C, et al. Chemotherapy and targeted therapy in adenoid cystic carcinoma of the head and neck: a review. Head \& neck. 2011:33(6):905-11. Epub 2010/07/24.

24. Iseli TA, Karnell LH, Graham SM, Funk GF, Buatti JM, Gupta AK, et al. Role of radiotherapy in adenoid cystic carcinoma of the head and neck. J Laryngol Otol. 2009;123(10):1137-44. Epub 2009/07/04.

\section{Submit your next manuscript to BioMed Central and we will help you at every step:}

- We accept pre-submission inquiries

- Our selector tool helps you to find the most relevant journal

- We provide round the clock customer support

- Convenient online submission

- Thorough peer review

- Inclusion in PubMed and all major indexing services

- Maximum visibility for your research

Submit your manuscript at www.biomedcentral.com/submit

C) Biomed Central 